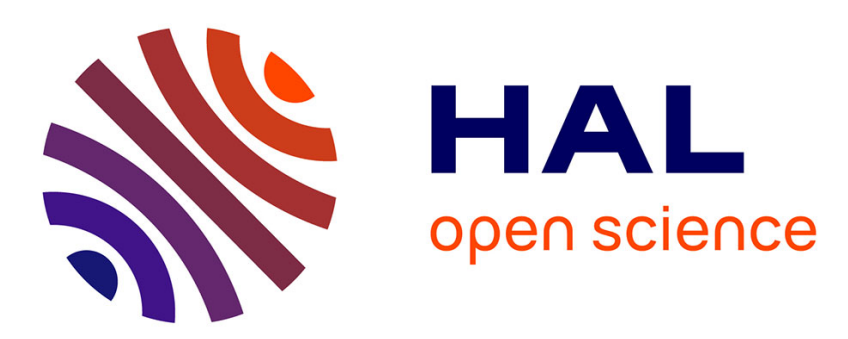

\title{
Study of the nonlinear stationary dynamic of single and multi-instabilities for disk brake squeal
}

Jean-Jacques Sinou, Nicolas Coudeyras, Samuel Nacivet

\section{To cite this version:}

Jean-Jacques Sinou, Nicolas Coudeyras, Samuel Nacivet. Study of the nonlinear stationary dynamic of single and multi-instabilities for disk brake squeal. International Journal of Vehicle Design, 2009, 51 (1-2), pp.207 - 222. 10.1504/IJVD.2009.027122 . hal-00411747

\section{HAL Id: hal-00411747 \\ https://hal.science/hal-00411747}

Submitted on 8 Feb 2013

HAL is a multi-disciplinary open access archive for the deposit and dissemination of scientific research documents, whether they are published or not. The documents may come from teaching and research institutions in France or abroad, or from public or private research centers.
L'archive ouverte pluridisciplinaire HAL, est destinée au dépôt et à la diffusion de documents scientifiques de niveau recherche, publiés ou non, émanant des établissements d'enseignement et de recherche français ou étrangers, des laboratoires publics ou privés. 


\title{
Study of the nonlinear stationary dynamic of single and multi instabilities for disc brake squeal
}

\author{
Jean-Jacques Sinou ${ }^{1}$, Nicolas Coudeyras ${ }^{1 \text { and } 2}$ and Samuel Nacivet ${ }^{2}$ \\ ${ }^{1}$ Laboratoire de Tribologie et Dynamique des Systèmes UMR CNRS 5513, Ecole \\ Centrale de Lyon, 36 avenue Guy de Collongue, 69134 Ecully cedex, France. \\ ${ }^{2}$ PSA Peugeot Citröen, 18 rue des Fauvelles, 92250 La Garenne Colombes, France. \\ Email : jean-jacques.sinou@ec-lyon.fr
}

\begin{abstract}
The focus of this article is to show and discuss the nonlinear dynamical behaviors of brake system subjected to friction-induced vibration that can be generated due to the co-existence of multi-unstable modes.

A finite element model with friction coupling is used to analyze the stability of the brake system and the stationary nonlinear oscillations for squeal noise prediction. The mechanism of squeal instability considers a mode coupling phenomenon that is classically referred to as coalescence.
\end{abstract}

Keywords: squeal, self-excited vibrations, periodic and quasi-periodic oscillations, nonlinear and harmonic components.

\section{Introduction}

One of the major challenges for vehicle manufacturers is the detection and prediction of squeal noise arising due to friction-induced vibrations in brake systems.

To study the squeal phenomenon in brake, the determination of the mechanism of the self-excited vibration is one of the first and most important phases to be discussed. As explained by Ibrahim [1-2], the first mechanisms that are based on friction characteristics and consider the variation of the friction coefficient are not sufficient to explain the occurrence of squeal. The second hypotheses in explaining the squeal phenomenon state that friction-induced vibrations are based on the structural dynamics properties and geometrical mode coupling. One of the first studies was presented by Spurr [3] with the sprag-slip model. Then, Earles and co-workers [4-5], and North [6] proposed various analytical models with two or more degrees-of-freedom that illustrated the importance of structural dynamics and demonstrated that the mode coupling can lead to onset of squeal instability. We refer the interested reader to the reviews [1-2,7-8] for an extensive overview of the different models and mechanisms of automotive disc brake squeal that are not the subject of this study. 
Even if the first models to study the squeal phenomenon were based on analytical models with few degrees-of-freedom, being generally sufficient to understand the problem of friction-induced vibrations, extensive studies using finite element method are actually performed to analyze brake squeal propensity [9-12].

So the aim of this paper is to investigate the squeal prediction and to clarify the nonlinear behaviors that might be observed by using a classical finite element model of brake system including the disc and the pad. The nonlinear dynamical behaviors of squeal noise are discussed in order to obtain some indications that might be useful in detecting and understanding the nonlinear friction-induced vibrations in brake system.

The study is set up as follows: first, the nonlinear finite element model of modecoupling instability in brake system is presented. Second, a brief review of the stability analysis and squeal prediction are given. Then, the nonlinear behaviors of squeal phenomenon due to a single mode coupling or multi mode couplings are presented and discussed. Different analyses are considered to provide information on the effects of multi-instabilities and the importance of the nonlinear harmonic components of the nonlinear vibrations in squeal prediction.

\section{Numerical model}

The system under study represents a simplified brake. It is composed by a pad and a disc that are the main components contributing to squeal. The pad and disc system without frictional interfaces are discretized by using a finite element approximation via Abaqus software, as shown in Figure 1. The caliper-piston assembly is not defined in this simplified brake. Hence the hydraulic pressure is directly applied to the backplate of the pad. The system is reduced by using a Craig and Bampton technique and keeping certain contact nodes (i.e. at the disc/pad interface) and retaining the first fifty eigenmodes of each component of the brake system.

The set of equation describing the dynamic system can be written by

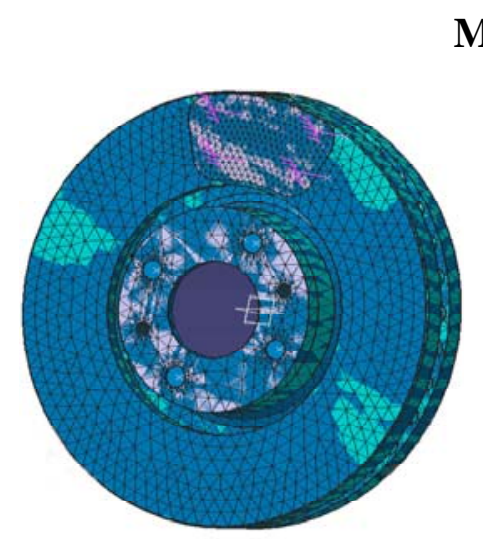

(a)

$$
\mathbf{M} \ddot{\mathbf{x}}+\mathbf{C} \dot{\mathbf{x}}+\mathbf{K x}=\mathbf{F}_{\mathrm{NL}}+\mathbf{F}_{\mathrm{ext}}
$$

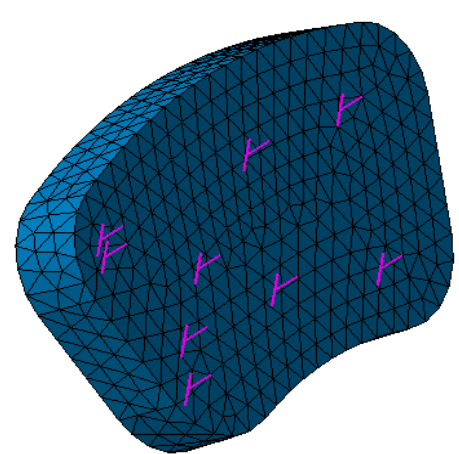

(b)

Figure 1: Finite element model (a) disc (b) pad 


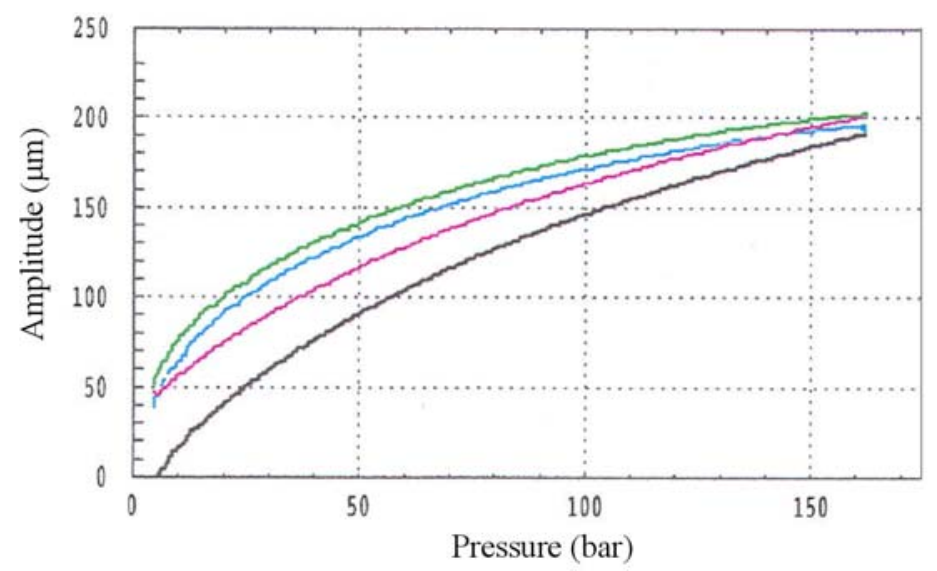

Figure 2: Experimental tests - pad compression

where $\mathbf{M}$ and $\mathbf{C}$, respectively, the mass and damping matrices. $\mathbf{K}$ represents the structural stiffness matrix. $\mathbf{x}$ is the displacement vector and dot denotes derivative with respect to time. $\mathbf{F}_{\mathbf{e x t}}$ is the vector force due to brake pressure applied on the pad. $\mathbf{F}_{\mathbf{N L}}$ contains the linear and nonlinear terms due to the friction contact between the pad and the disc.

The friction interface is modelled by using dedicated software in Matlab and by introducing in the finite element model the nonlinear contact force vectors that are defined by

$$
\begin{aligned}
& F_{\text {contact }, X}^{p}= \begin{cases}k_{l}\left(x_{p}-x_{d}\right)+k_{n l}\left(x_{p}-x_{d}\right)^{3} & \text { if }\left(x_{p}-x_{d}\right)>0 \\
0 & \text { otherwise }\end{cases} \\
& F_{\text {contact }, X}^{d}= \begin{cases}-k_{l}\left(x_{p}-x_{d}\right)-k_{n l}\left(x_{p}-x_{d}\right)^{3} & \text { if }\left(x_{p}-x_{d}\right)>0 \\
0 & \text { otherwise }\end{cases} \\
& F_{\text {contact }, Y}^{p}=\mu F_{\text {contact }, X}^{p} \operatorname{sign}\left(v_{r}\right) \\
& F_{\text {contact }, Y}^{d}=\mu F_{\text {contact }, X}^{d} \operatorname{sign}\left(v_{r}\right)
\end{aligned}
$$

where "d" denotes the disc and "p" the pad. The displacements $x_{d}$ and $x_{p}$ are defined in the direction normal to the contact surface; then $F_{\text {contact }, Y}$ and $F_{\text {contact }, X}$ are the contact forces along the tangential and normal directions respectively. $\mu$ is the friction coefficient that is assumed to be constant and the classical Coulomb's law is applied. $v_{r}$ defines the relative velocity between the pad and disc. $k_{l}$ and $k_{n l}$ correspond to the linear and nonlinear stiffnesses contact respectively. This formulation has been chosen 
to fit the first and the third order of pad compression curves obtained from experimental tests, as showed in Figure 2. As indicated in the previous equations, contact and nocontact configurations at the friction interface are possible so that the pad and disc can separate locally or can open and close repeatedly at some local nodes during the vibration.

\section{Stability analysis}

The complex eigenvalue analysis is the first tool to provide the physical parameters that lead to instabilities of the brake system and the squeal occurrences.

The stability of the nonlinear system is calculated by considering the linearized system at the equilibrium point. This static equilibrium position $\mathbf{x}_{\mathbf{0}}$ is obtained by solving the nonlinear static equations that satisfy the following conditions:

$$
\mathbf{K} \mathbf{x}_{0}=\mathbf{F}_{\mathrm{NL}}+\mathbf{F}_{\mathrm{ext}}
$$

Stability of the static position $\mathbf{x}_{\mathbf{0}}$ is then investigated for a small perturbation $\overline{\mathbf{x}}$ around the equilibrium point:

$$
\mathbf{x}=\mathbf{x}_{\mathbf{0}}+\overline{\mathbf{x}}
$$

After calculation, the linearized system is given by

$$
\mathbf{M} \ddot{\overline{\mathbf{x}}}+\mathbf{C} \dot{\overline{\mathbf{x}}}+\left(\mathbf{K}-\mathbf{K}_{\mathbf{L}}\left(\mathbf{x}_{\mathbf{0}}\right)\right) \overline{\mathbf{x}}=\mathbf{0}
$$

where $\mathbf{K}_{\mathbf{L}}$, which is asymmetric, represents the linearized expression of the nonlinear frictional contact $\mathbf{F}_{\mathrm{NL}}$ at the equilibrium position

$$
\left.\mathbf{F}_{\mathrm{NL}}(\overline{\mathbf{x}}) \approx \sum_{i} \frac{\partial \mathbf{F}_{\mathrm{NL}}(\overline{\mathbf{x}})}{\partial \bar{x}_{i}}\right|_{\mathbf{x}_{0}} \bar{X}_{i}=\mathbf{K}_{\mathbf{L}}\left(\mathbf{x}_{\mathbf{0}}\right) \overline{\mathbf{x}}
$$

So the local stability around the equilibrium point $\mathbf{x}_{\mathbf{0}}$ is investigated by calculating the eigenvalues of the Jacobian matrix $\mathbf{J}$

$$
\mathbf{J}=\left[\begin{array}{cc}
\mathbf{0} & \mathbf{I} \\
-\mathbf{M}^{-1}\left(\mathbf{K}-\mathbf{K}_{\mathbf{L}}\left(\mathbf{x}_{\mathbf{0}}\right)\right) & -\mathbf{M}^{-1} \mathbf{C}
\end{array}\right]
$$

The complex eigenvalues $\lambda$ can be expressed as $\lambda=a+i b$ where the real part $a$ indicates the stability of the equilibrium point and $b$ represents the pulsation of the mode. As long as the real part of all the eigenvalues remains negative, the equilibrium point of the system is stable. When at least one of the eigenvalues has a positive real part, the equilibrium point is unstable and squeal noise occurs.

Figures 3 display the evolution of real parts and frequencies as a function of the friction coefficient. For a friction coefficient $\mu$ equal to zero, the real parts of eigenvalues are 
negative due to the structural damping introduced in the system; so, the system features stable separate modes in the frequency range.

As the friction coefficient increases, Figure 3(b) illustrates that the real parts increase slowly. The associated frequencies around $1510 \mathrm{~Hz}$ tend to get closer, as indicated in Figure 3(a). The Hopf bifurcation point occurs for a friction value of 0.26 (see Figure 3(b)). Showing Figure 3(a), it can be noted that the two modes do not reach exactly the same frequency at the bifurcation point due to a non-equally damped configuration between the two modes (see the paper of Fritz et al. [12] for more details). For the reader comprehension, the red lines in Figures 3 correspond to the unstable mode. This phenomenon is classified as a non-perfect coalescence. This non-perfect coalescence can also be seen by considering the gap in real part between the two modes at $\mu=0$. As explained by Hoffmann and Gaul [13] and Fritz et al. [14], a "smoothing effect"' of the curves with respect to the friction coefficient is observed, both for real parts and frequencies in the case of non-equally damped configuration.

After the first Hopf bifurcation point at $\mu=0.26$, a second instability appears when the value of the friction coefficient is greater than 0.28 (see Figure 3(b) where the continues and dotted lines are associated with the frequencies at $920 \mathrm{~Hz}$ and $1510 \mathrm{~Hz}$, respectively). The upper Figure 3(a) illustrates the coalescence phenomena around $920 \mathrm{~Hz}$. Finally, Figures 4 show the evolutions of both real parts and frequencies with increasing the friction coefficient for the two instabilities (at $920 \mathrm{~Hz}$ and $1510 \mathrm{~Hz}$ ). It can be observed that one of the two modes that coalesce is stable whereas the other one is unstable for the two instabilities. However, for the first instability (at $1510 \mathrm{~Hz}$ ), the unstable mode corresponds to the higher mode, whereas for the second instability (at $920 \mathrm{~Hz}$ ), the unstable mode corresponds to the lower mode. In these two cases, the less damped eigenvalue becomes unstable.
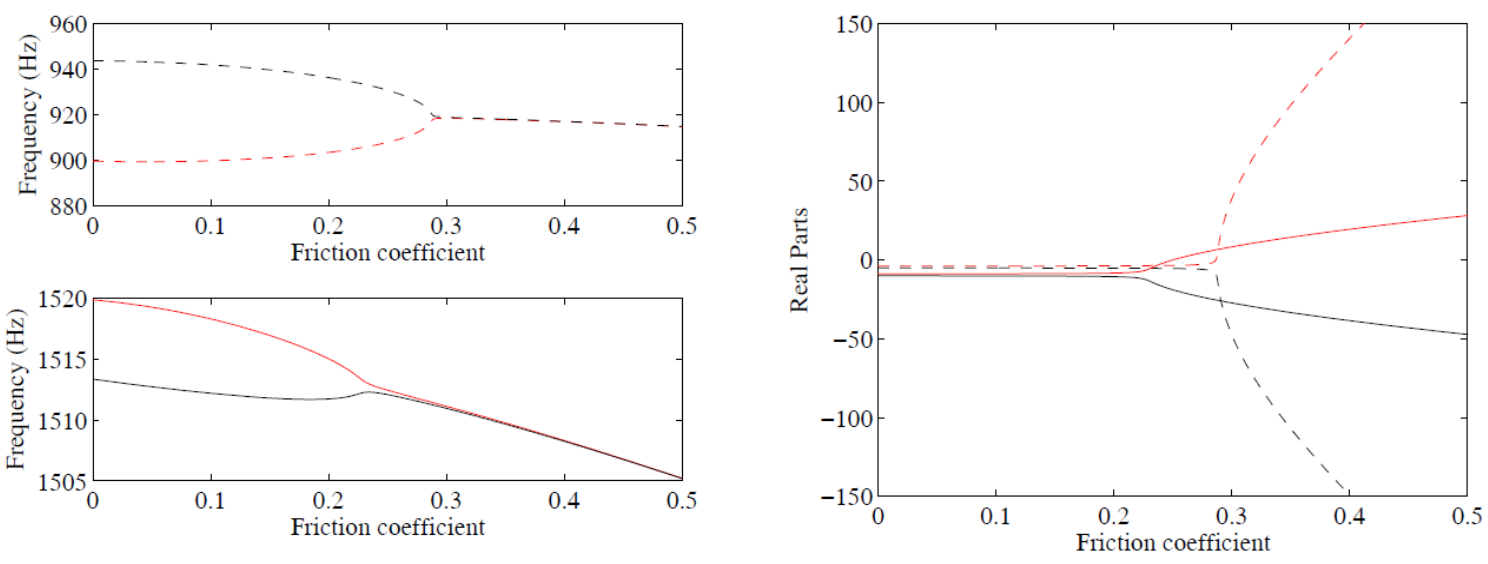

(a)

(b)

Figure 3 : coalescences patterns $(\mathrm{a})$ frequency (b) real parts (red lines = unstable modes) 


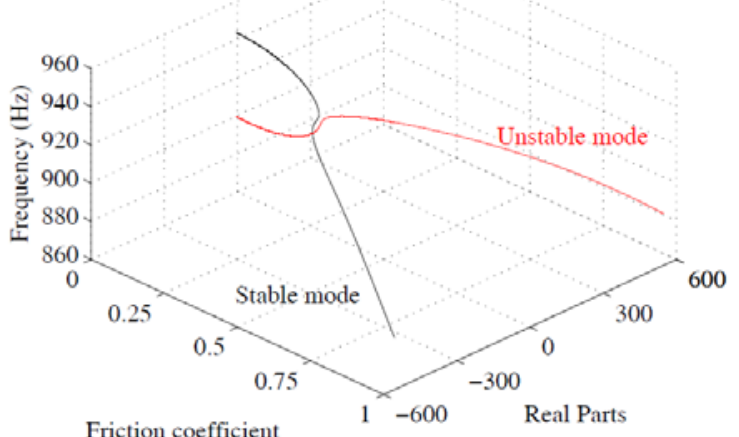

(a)

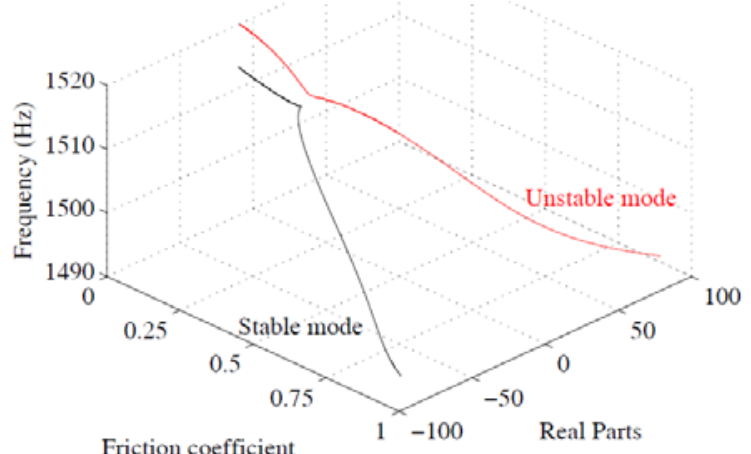

(b)

Figure 4 : 3Dplot of the coalescence patterns (a) at $920 \mathrm{~Hz}$ (b) at $1510 \mathrm{~Hz}$

\section{Nonlinear self-excited vibrations}

Even if the stability analysis is the first step for designing mechanical systems subjected to friction-induced vibration, the determination of the vibrational amplitudes is essential for a robust design of brake systems. It can be noted that a brake system may be considered to be efficient if the self-excited vibrations do not become excessive and so no offensive noises are generated. In other words, a brake system with small selfexcited oscillations but performing properly can be validated in a design process. So not only the stability analysis but also the amplitudes and the vibrational behaviour of the nonlinear oscillations are key factors.

The time-history responses of the nonlinear dynamic system can be obtained by solving the original full nonlinear equation via a numerical integration. Here, the nonlinear system is rewritten in state variables and variable order Adams-Bashforth-Moulton PECE solver is used.

In this section, specific cases (for various friction coefficients) will be given to illustrate the different nonlinear behaviour of the brake system that can be observed during squeal vibrations. The first case corresponds to self-excited oscillations of the brake system with a "single instability" participation (with only one unstable mode). It represents one of the most classical nonlinear behavior observed during squeal phenomenon. The second one illustrates the "multi-instabilities" case when the nonlinear self-excited vibrations appear due to the presence of two unstable modes.

\subsection{Single instability}

Firstly, Figures 5(a) illustrate the nonlinear oscillations of the pad (Y-component) in the time domain for the friction coefficient $\mu=0.26$ when only one instability is present (as previously seen in Section 2). The displacement and velocity growth leading to periodic oscillations is one of the most typical nonlinear features of the squeal 
phenomenon. Figure 5(b) shows the associated limit cycle amplitudes. Moreover, Figures 6 illustrate the limit cycle amplitudes of the pad in the $\mathrm{X}$ and $\mathrm{Z}$ directions.

\begin{tabular}{ccc}
\hline Friction coefficient & Frequency $\mathrm{f}_{1}(\mathrm{~Hz})$ & Frequency $\mathrm{f}_{2}(\mathrm{~Hz})$ \\
\hline 0.26 & - & 1512 \\
0.28 & 897 & 1497 \\
0.35 & 904 & 1495 \\
0.4 & 907 & - \\
\hline
\end{tabular}

Table 1: Fundamental frequencies of the nonlinear responses
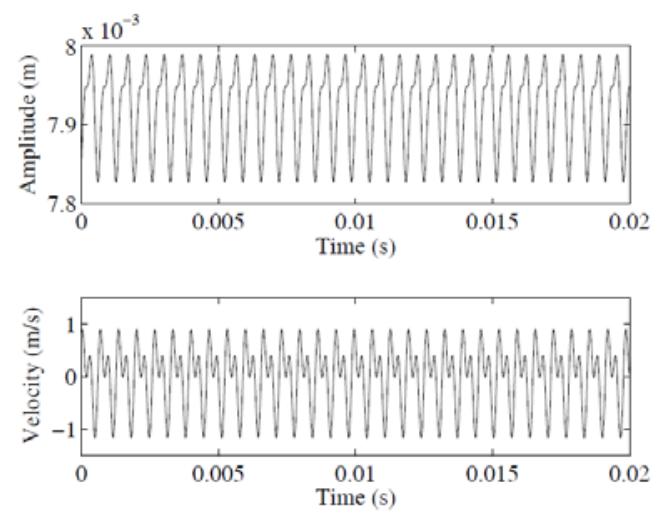

(a)

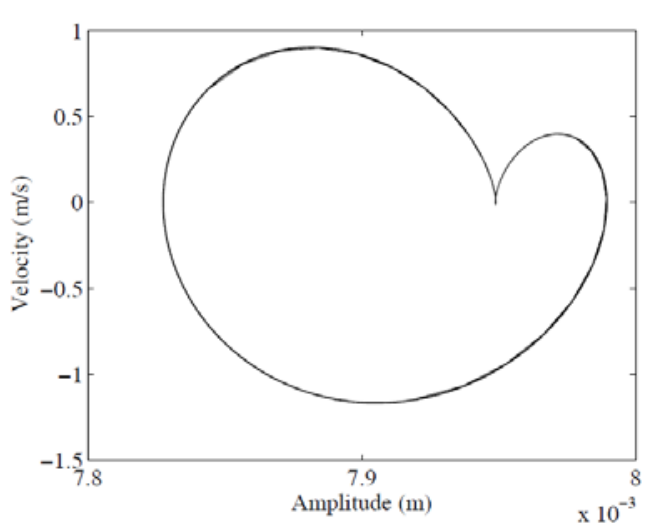

(b)

Figure 5 : stationary response of the Y-component of the pad for $\mu=0.26$ (a) zoom on the displacement and velocity (b) limit cycles

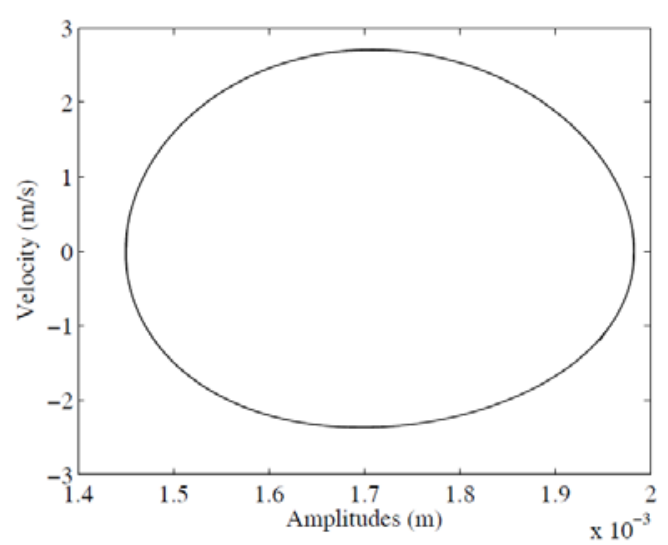

(a)

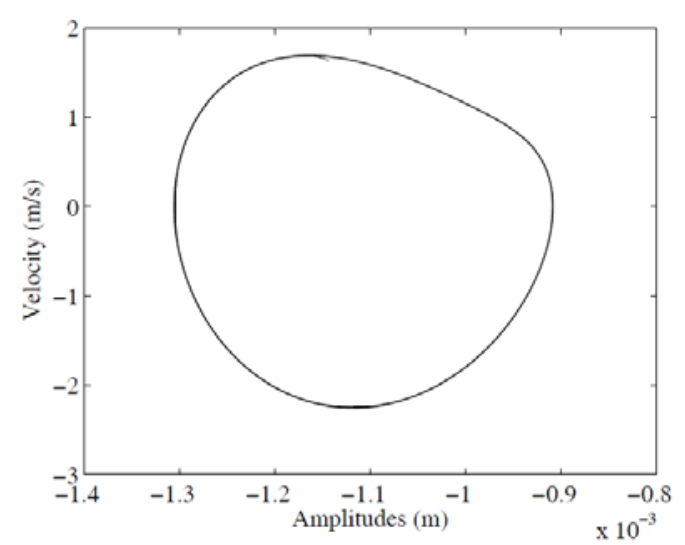

(b)

Figure 6 : Limit cycles of the pad for $\mu=0.26$ (a) X-direction (b) Z-direction 
The spectrum components of the nonlinear self-excited amplitudes are analyzed by using the Fast Fourier Transformation method to derive the Power Spectrum Density of the displacement. Results for a node of the pad (in the X,Y and Z directions) are plotted in Figure 7: the fundamental frequency that corresponds to the unstable frequency of the mode around $1512 \mathrm{~Hz}$ (as previously showed by the eigenvalues analysis) and its second and third harmonics with decreasing amplitudes are present.

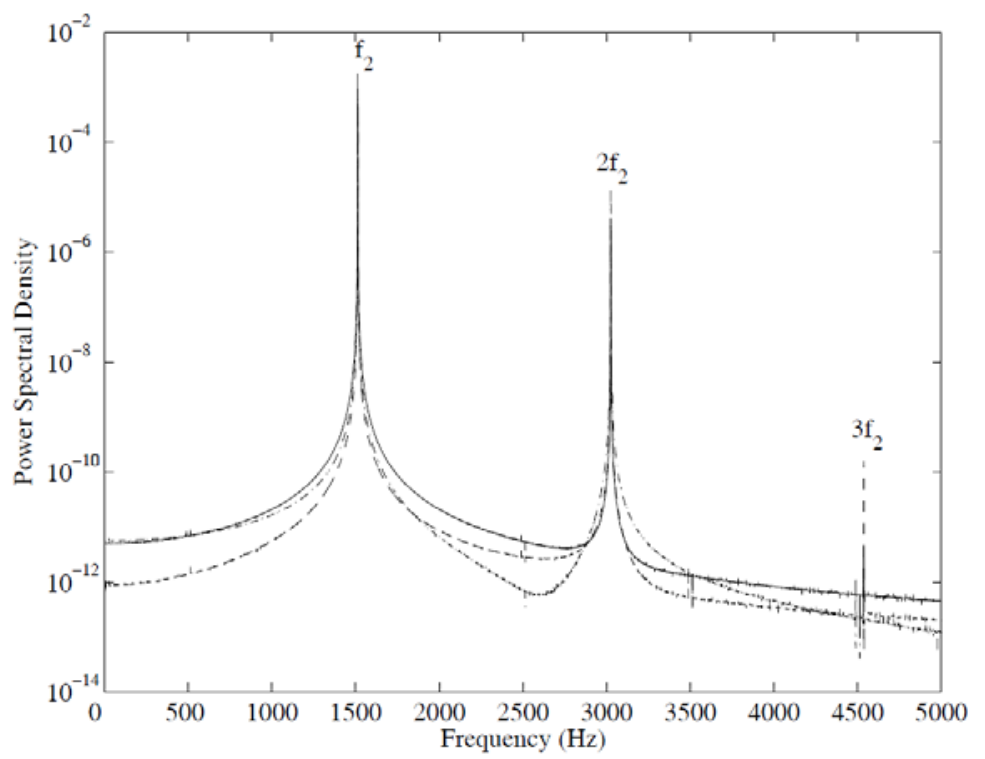

Figure 7 : Power Spectral Density of the stationary response of the pad for $\mu=0.26$ ( - X-direction,-- - Y direction,-- Z-direction)

The contribution of the $1 \times$ and $2 \times$ harmonic components are also clearly shown on the limit cycles with the small "double-inside loops" that characterizes the presence of the second order for the complete nonlinear response of mechanical systems (see Figure 5(b)). The contribution of the third order in the nonlinear response can be identified in the spectrum (see Figure 7). However the contribution of the $3 \times$ harmonic components is less significance than the $1 \times$ and $2 \times$ harmonic components. In view of the above, it can be concluded that the nonlinear self-excited oscillations during the "single squeal instability" is composed by harmonic oscillations.

The nonlinear time simulation agrees with the instability predictions of the possible unstable modal coupling. In fact, not only the fundamental component of the unstable mode but also contributions of the second and third orders are detected in periodic oscillations of the squeal signal. So the nonlinear contributions and the associated harmonic frequency components must be taken into account not only for predicting the complete nonlinear periodic vibration of the "single squeal" instability but also for avoiding worse designs that could neglect the presence of extra harmonic resonant peaks of the fundamental unstable mode. Then, it is observed that the fundamental 
frequency of the stationary self-excited oscillations is not exactly those of the unstable mode obtained by using eigenvalues analysis, as indicated in Table 1.

Finally, it can be noted that the unstable conditions, the limit cycle amplitudes and the associated self-excitation mechanism have been obtained even if a constant friction coefficient is used in the numerical simulation. As reported above, the present work deals with squeal phenomenon as a modal instability.

\subsection{Multi-instabilities}

In this section, the nonlinear behaviour of the brake system subjected to multi squeal instabilities is investigated.

Figure 8(a) shows the quasi-periodic oscillations of the pad (Y-component) for the friction coefficient $\mu=0.28$. This value of the friction coefficient corresponds to the appearance of the second instability, as previously indicated in Section 3. The associated limit cycle is given in Figure 8(b). Moreover, the limit cycles of the pad in the $\mathrm{X}$ and Z-directions are plotted in Figures 9. It clearly appears that the nonlinear behaviour of the brake system is complex due to the contributions of the two instable modes.

Figure 10 shows the Power Spectrum Density of the three previous components of the pad (X,Y and $\mathrm{Z}$ components): in this case, it is observed that the fundamental frequencies in the spectrum are the frequency $f_{2}$ of the first squeal instability (at $1497 \mathrm{~Hz}$ ) and the frequency $\mathrm{f}_{1}$ of the second squeal instability (at $897 \mathrm{~Hz}$ ). The second and third harmonics of the second instability (i.e. $2 \mathrm{f}_{1}, 3 \mathrm{f}_{1}$ ) and the second harmonics of the first instability (i.e. $2 \mathrm{f}_{2}$ ) are also identified. Moreover, it clearly appears that the two unstable modes $f_{1}$ and $f_{2}$ interact together to product sum and difference frequencies $f_{2^{-}}$ $f_{1}$ and $f_{2}+f_{1}$. The component $f_{2}-f_{1}$ is prominently seen, being equal to $f_{2}$. The spectrum hence indicates the combination harmonics such as $2 f_{1}-f_{2}, 3 f_{1}-f_{2}, 2 f_{2}-f_{1}, f_{2}+f_{1}, 2 f_{1}+f_{2}$, etc.
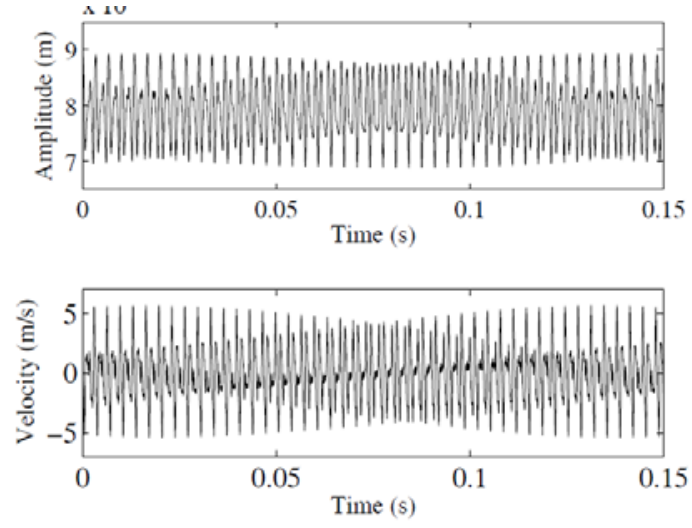

(a)

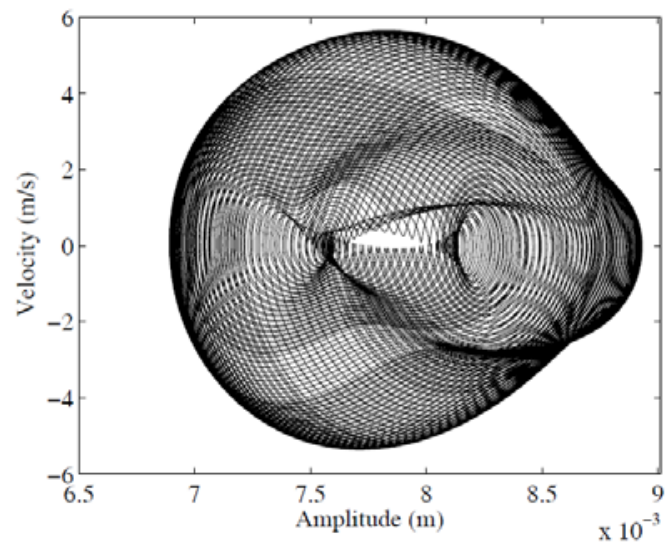

(b)

Figure 8 : stationary response of the pad (Y-component) for $\mu=0.28$ (a) zoom on the displacement and velocity (b) limit cycles 


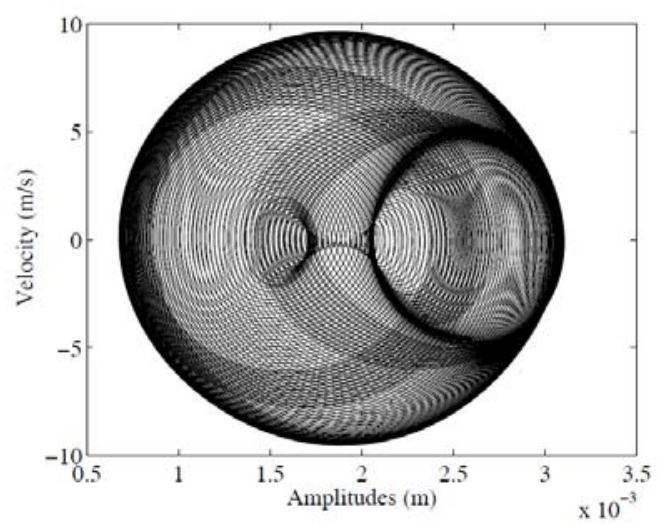

(a)

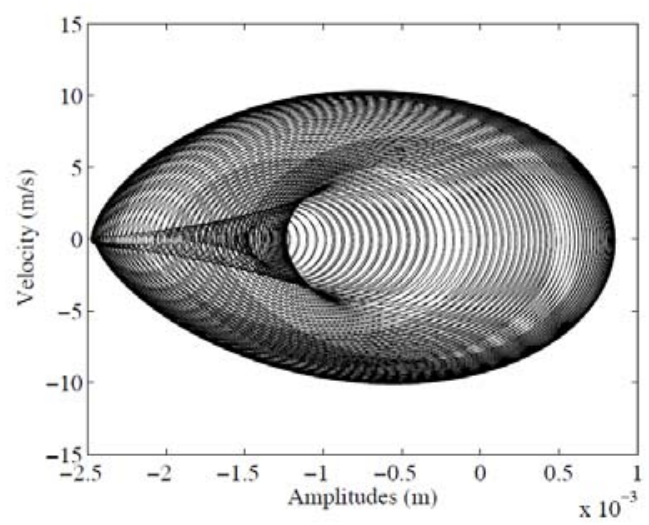

(b)

Figure 9 : limit cycles of the pad for $\mu=0.28$ (a) X-direction (b) Z-direction

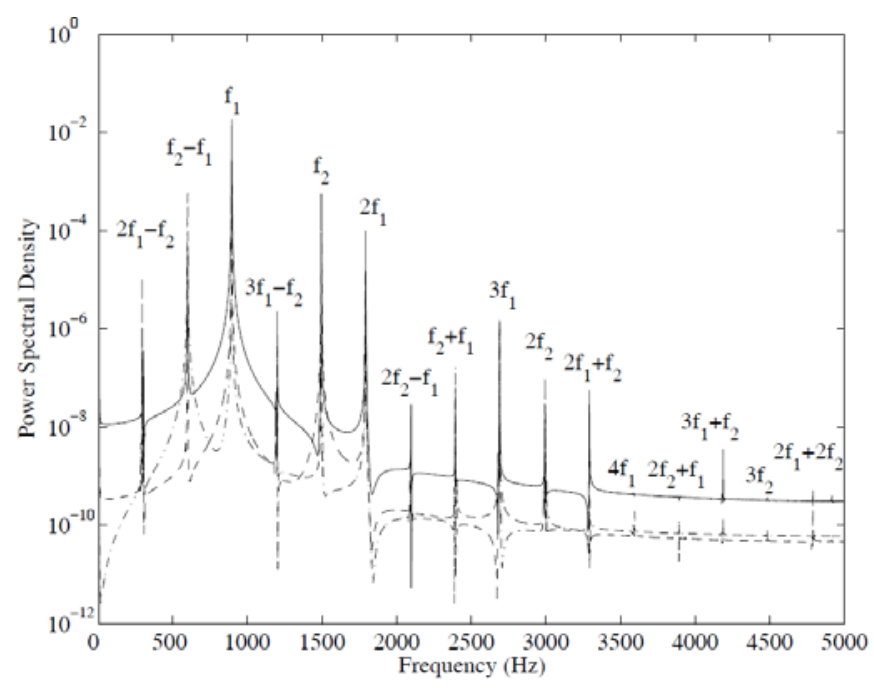

Figure 10 : Power Spectral Density of the stationary response of the pad for $\mu=0.28$ ( X-direction, - $¥$ direction, -Z-direction)

Even if these combination of frequency components are less important than the fundamental frequencies $\left(f_{1}\right.$ and $\left.f_{2}\right)$, its harmonics $\left(2 f_{1}, 3 f_{1}\right.$ and $\left.2 f_{2}\right)$, and the sum and difference frequencies $\left(f_{2}-f_{1}\right.$ and $\left.f_{2}+f_{1}\right)$, they are prominently seen in the spectrum. The presence of the combination harmonics of the two instabilities is indicative of strong participation and "coupling" of the two unstable modes that leads to quasi-periodic selfexcited oscillations of the brake system. Finally, other combinations $\left(4 f_{1}, 3 f_{2}, f_{2}+f_{1}\right.$, $2 \mathrm{f}_{2}+2 \mathrm{f}_{1}$ and $3 \mathrm{f}_{1}+\mathrm{f}_{2}$ ) of less significance could be detectable, as indicated in Figure 10. 

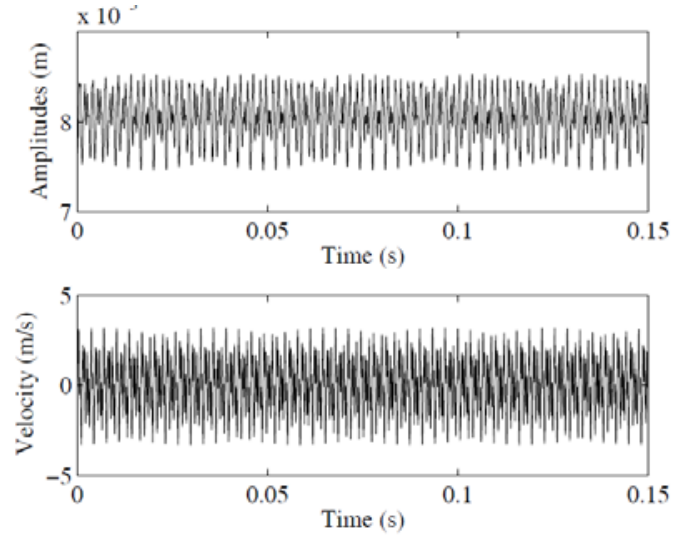

(a)

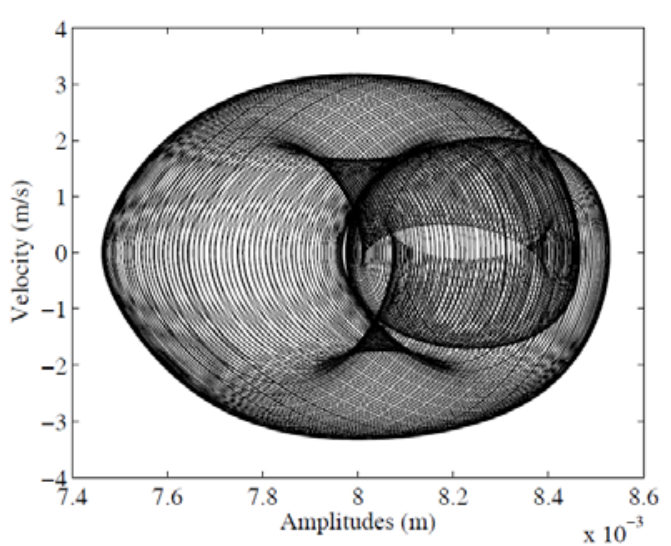

(b)

Figure 11 : stationary response of the pad (Y-component) for $\mu=0.35$ (a) zoom on the displacement and velocity (b) limit cycles

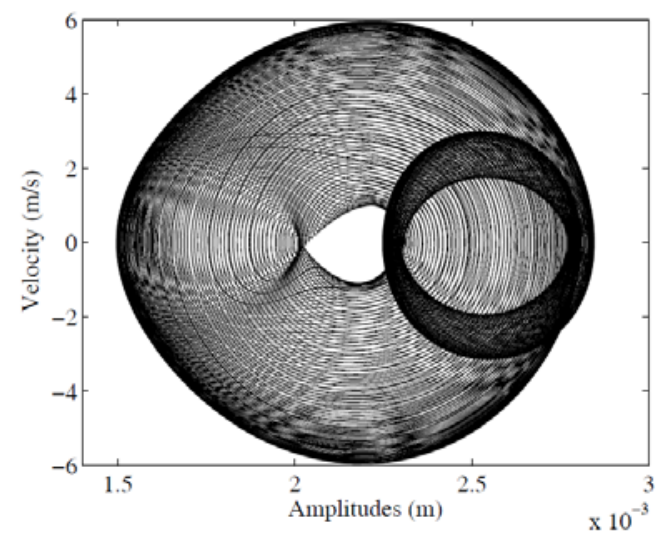

(a)

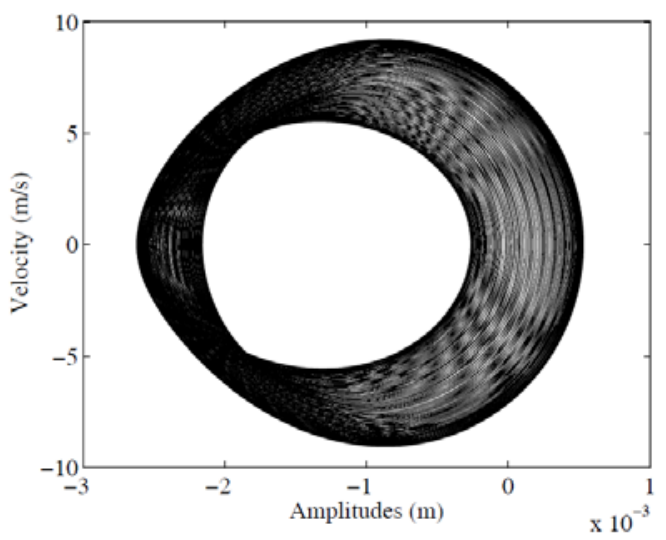

(b)

Figure 12 : limit cycles of the pad for $\mu=0.35$ (a) X-direction (b) Z-direction

Then Figures 11 illustrate the nonlinear oscillations of the pad (Y-component) and the associated limit cycle for the friction coefficient $\mu=0.35$. Figures 12 show the limit cycles of the pad in the $X$ and $Z$ directions. As previously shown (for $\mu=0.28$ ), a complex nonlinear vibrational behavior is observed. It can be noted that the amplitudes of the pad in this case are smaller than the amplitudes for $\mu=0.28$. So increasing the friction coefficient does not imply an increase of the limit cycles amplitudes of the brake system.

Figure 13 shows the Power Spectrum Density for the friction coefficient $\mu=0.35$ for the $\mathrm{X}, \mathrm{Y}$ and $\mathrm{Z}$ components of the pad. The fundamental frequencies in the spectrum of this case are the frequency $\mathrm{f}_{2}$ of the first squeal instability (at $1495 \mathrm{~Hz}$ ) and the frequency $\mathrm{f}_{1}$ of the second squeal instability (at $904 \mathrm{~Hz}$ ). In comparison with the previous case 
( $\mu=0.28$ ), it is observed that the frequency of the first squeal instability decreases whereas the frequency of the second instability increases (see Table 1).

The second and third harmonics of the second instability (i.e. $2 \mathrm{f}_{1}, 3 \mathrm{f}_{1}$ ) are important whereas the second harmonics of the first instability (i.e. $2 \mathrm{f}_{2}$ ) are less significant. Then, interactions between the two unstable modes $f_{1}$ and $f_{2}$ are clearly identified: the combination harmonics such as $2 \mathrm{f}_{1}-\mathrm{f}_{2}$ and $\mathrm{f}_{2}-\mathrm{f}_{1}$ are prominently observed, being equal to $2 \mathrm{f}_{1}$ or $3 \mathrm{f}_{1}$.

Other combination harmonics of less significance that have been previously observed for $\mu=0.28$ are also present for $\mu=0.35$, such as $3 f_{1}-f_{2},-f_{1}+f_{2}, 2 f_{1}+f_{2}, 2 f_{2}-f_{1}, 4 f_{1}, 3 f_{1}+f_{2}$, $2 f_{2}+f_{1}, 2 f_{1}+2 f_{2}$. It is noted that the participation of these combination harmonics are practically identical for the two cases.

Finally, the presences of new combination harmonics of the two instabilities are shown $\left(5 f_{1}-3 f_{2},-3 f_{1}+2 f_{2},-2 f_{1}+2 f_{2}, 5 f_{1}-2 f_{2}, 4 f_{1}-f_{2}, 5 f_{1}-f_{2}, 6 f_{1}-f_{2}, 5 f_{1}\right.$ and $\left.7 f_{1}-f_{2}\right)$. They are of less significance but they are clearly seen in the spectrum and so they indicate a strong participation and coexistence of the two unstable modes of fundamental frequencies $\mathrm{f}_{1}$ and $\mathrm{f}_{2}$ that leads to bi-periodic self-excited oscillations. Then, even if some combination harmonics appear to be very close (see for example $-3 f_{1}+2 f_{2}$ and $2 f_{1}-f_{2},-2 f_{1}+2 f_{2}$ and $3 f_{1}-$ $f_{2}$, or $2 f_{2}$ and $5 f_{1}-f_{2}$ ), they are clearly distinguished. It can be noted that these new combination harmonics correspond to the upper orders of the sum and difference frequencies $\pm \mathrm{nf} 1 \pm \mathrm{mf}_{2}$ (with $\mathrm{n}$ and $\mathrm{m}$ integers).

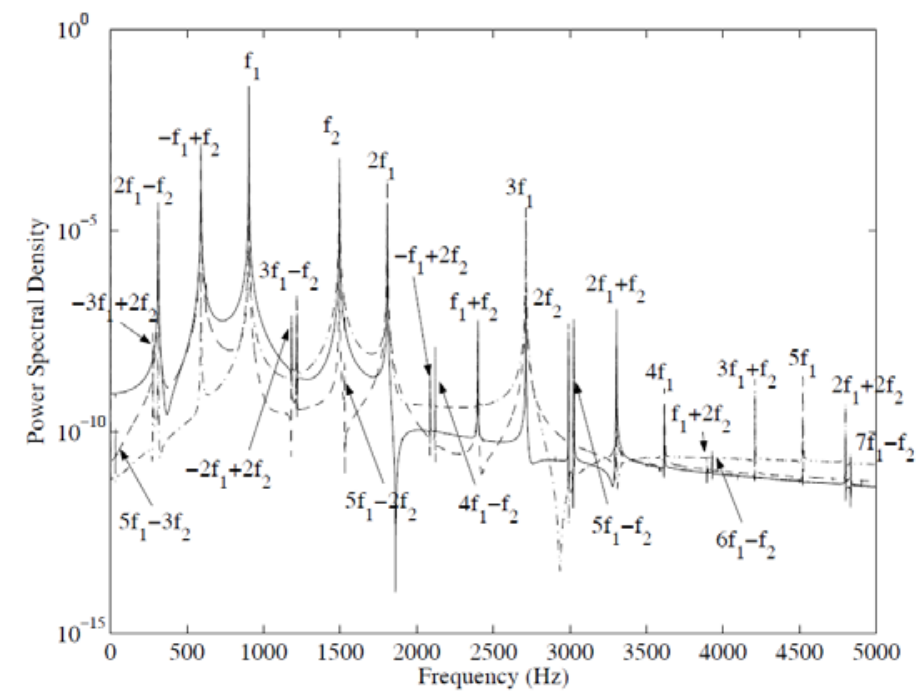

Figure 13 : Power Spectral Density of the stationary response of the pad for $\mu=0.35$ ( - X-direction, --Y direction,---Z-direction) 
Now, Figures 14, 15 and 16 show the nonlinear oscillations of the pad, the associated limit cycles and the Power Spectrum Density for the friction coefficient $\mu=0.4$ in the $\mathrm{X}, \mathrm{Y}$ and $\mathrm{Z}$ directions.

In this last case, the fundamental frequency $\mathrm{f}_{1}$ of the first unstable mode and its second and third harmonics components $2 \mathrm{f}_{1}$ and $3 \mathrm{f}_{1}$ are predominant. The typical "inside loop" of the limit cycle is shown in Figures 14(b) and 15(a). Contributions of less significance that corresponds to the $n \times$ harmonic components (for $n=4$ to 11 ) are also detected in the spectrum, as indicated in Figure 16.

Here, the presence of the first instability and the sum and difference frequencies or harmonics combinations of the two unstable modes are found to be absent in the spectrum. Hence, the nonlinear behavior of the self-excited system is similar to periodic oscillations and so appears more simple than the two previous cases (for the friction coefficient $\mu=0.28$ or $\mu=0.35$ ).

So, it may be concluded that even if the eigenvalues analysis indicates the presence of two instabilities, the second unstable mode of frequency $f_{2}$ does not participate in the nonlinear oscillations of the self-excited vibrations. One general explanation can be that the first instability of frequency $\mathrm{f}_{1}$ governs the nonlinear oscillations of the brake system hiding or "removing" the contributions of the second instability of frequency $\mathrm{f}_{2}$. As reported in the previous section, the fundamental frequencies of the self-excited oscillations are not exactly those obtained by using eigenvalues analysis, as indicated in Table 1.
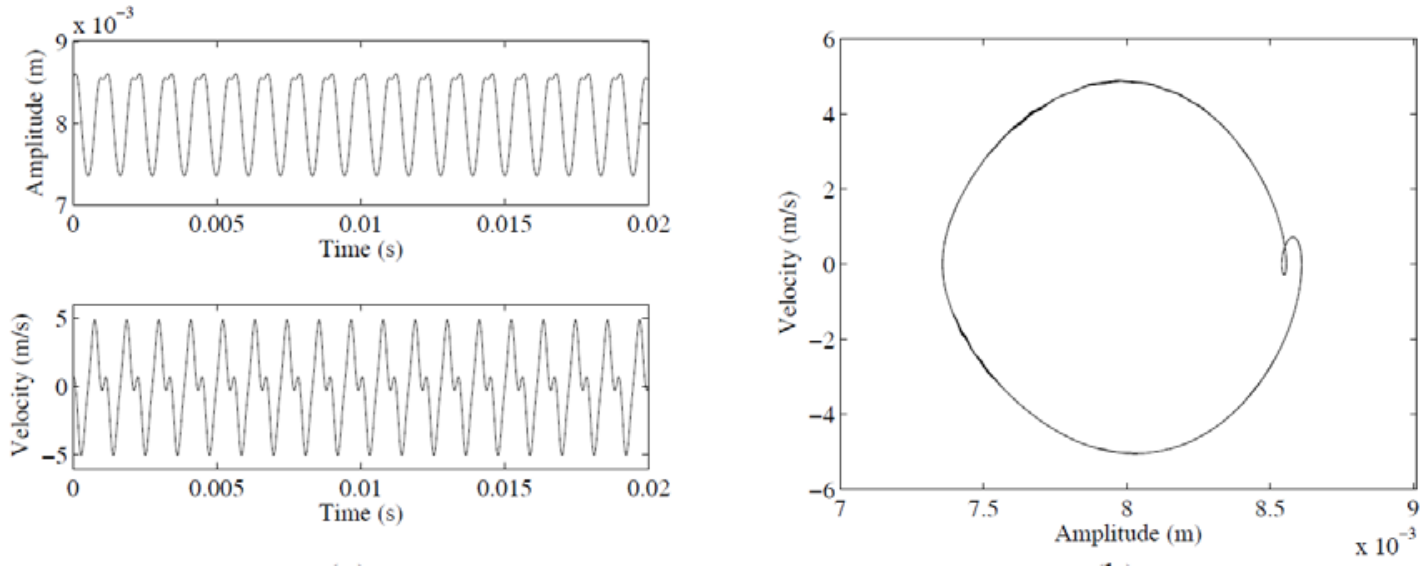

(a)

(b)

Figure 14 : stationary response of the pad (Y-component) for $\mu=0.4$ (a) zoom on the displacement and velocity (b) limit cycles 


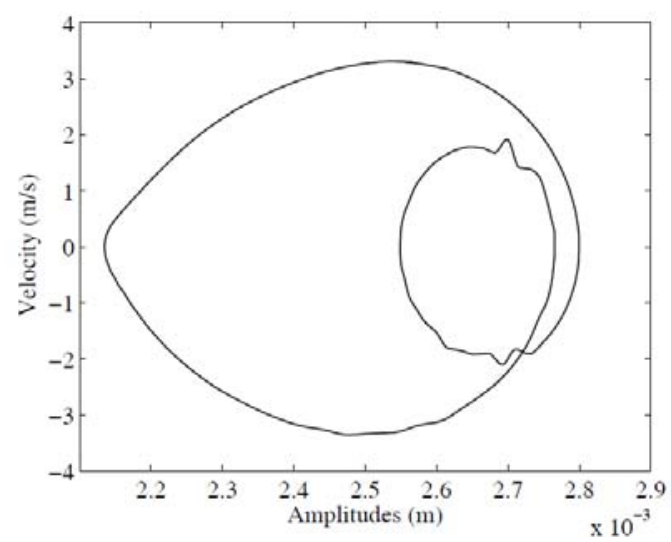

(a)

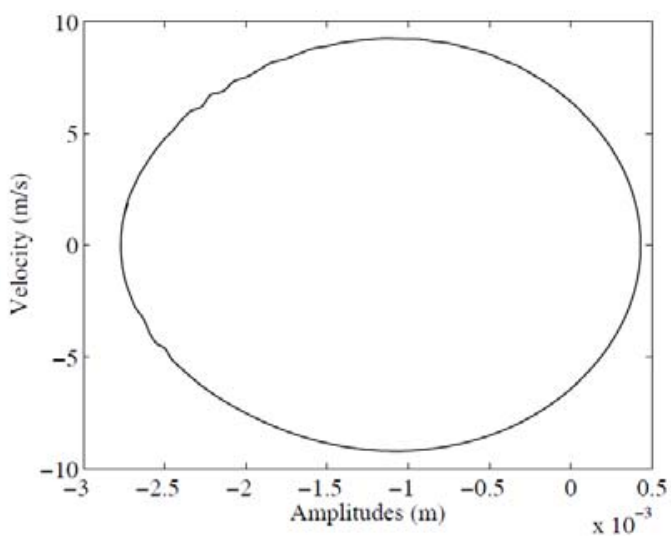

(b)

Figure 15 : limit cycles of the pad for $\mu=0.4$ (a) X-direction (b) Z-direction

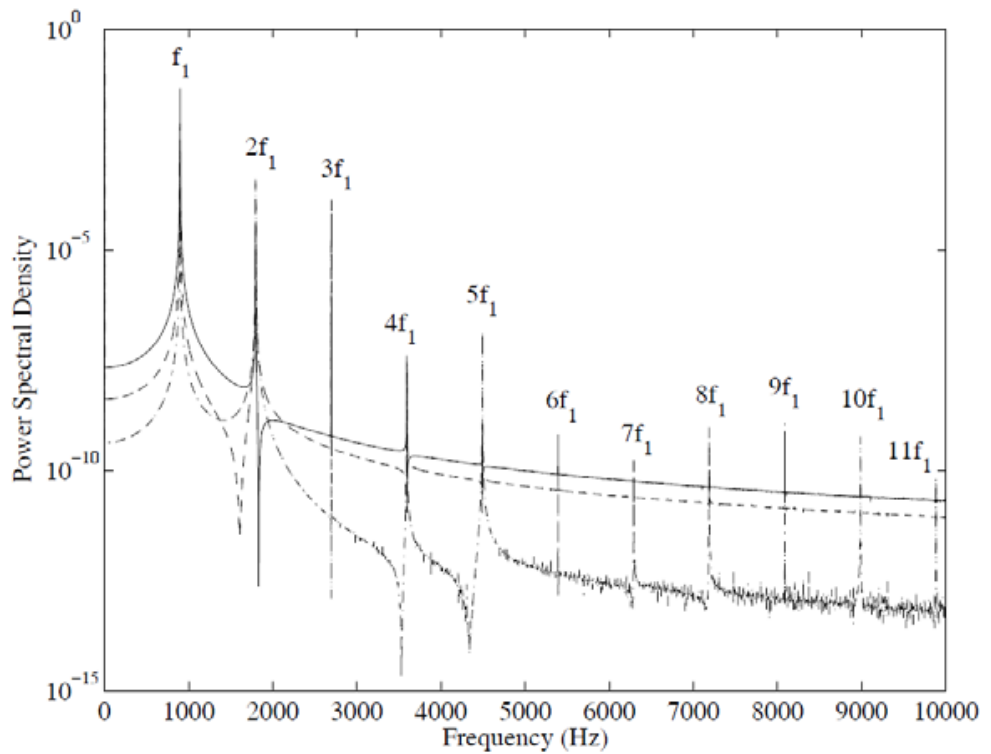

Figure 16 : Power Spectral Density of the stationary response of the pad for $\mu=0.4$ ( -X-direction, --Y direction, ---Z-direction)

\section{Conclusion}

In this study, we investigated numerically the nonlinear behaviour of disc brake squeal by using a simplified finite element model.

Firstly, two classical results are observed:

- when only one instability occurs in the brake system, the fundamental frequency and its harmonic are present in the nonlinear self-excited vibrations. The 
fundamental frequency of the nonlinear oscillations is not exactly the same than those obtained by applying eigenvalues analysis.

- The resonance peaks due to the fundamental frequencies component are usually greater than the resonance peaks due to the harmonics components or combinations of frequency components. However, the amplitudes of the harmonics components, sum and difference frequencies can not be neglected and clearly contribute to the self-excited oscillations of the brake system.

Secondly, two additional observations are obtained:

- The resonances of the nonlinear self-excited vibrations that satisfy the relationships $\pm \mathrm{nf} 1 \pm \mathrm{mf}_{2}$ (with $\mathrm{n}$ and $\mathrm{m}$ integers) occur due the coexistence of two instabilities of fundamental frequencies $f_{1}$ and $f_{2}$, respectively. So the amplitudes of peaks that do not correspond to the fundamental frequencies of unstable modes do not indicate the presence of new instability but show the harmonics of these frequencies that can contribute to the overall vibration.

- Even if the stability analysis indicates the existence of two instabilities, the nonlinear behaviour can be governed by only one unstable mode without a significant contribution of the other instability. As a result, the nonlinear selfexcited oscillations are only composed by the fundamental frequency $f_{1}$ or $f_{2}$ and its harmonics. So a method for the suppression of brake squeal should be to introduce energy to an other frequency that is out of the audible range (see for example the application of the Dither mechanism [15]).

In conclusion, the nonlinear self-excited vibrations of disc brake squeal can be very complex due to the presence of multi resonance peaks. Therefore, it becomes easier to avoid a worse design by utilizing not only the fundamental frequencies but also the harmonic components and the combination of frequencies components.

\section{Acknowledge}

The first author gratefully acknowledges the financial support of the french National Research Agency through the programme Young researcher ANR-07-JCJC-0059-01CSD 2.

\section{References}

[1] Ibrahim R.A., Friction-induced vibration, chatter, squeal and chaos: Part Imechanics of contact and friction, American Society of Mechanical Engineers Applied Mechanics Review, 47 (7), 209-226, 1994.

[2] Ibrahim R.A., Friction-induced vibration, chatter, squeal and chaos: Part IIdynamics and modeling, American Society of Mechanical Engineers Applied Mechanics Review, 47 (7), 227-253, 1994.

[3] Spurr, R. T.,. A theory of brake squeal. Proc. Auto. Div. Instn. Mech. Engrs, No. 1, 33-40. 1961-1962.

[4] Earles S.W.E., Lee C.K., Instabilities arising from the frictional interaction of a pin-disc system resulting in noise generation, Transactions of American Society of 
Mechanical Engineers Journal of Engineering for Industry Series B, 98 (1), 8186, 1976.

[5] Earles S.W.E, Chambers P.W., Disc brake squeal noise generation: predicting its dependency on system parameters including damping, International Journal of Vehicle Design, 8 (4-6), 538-552, 1987.

[6] North M.R., A mechanism of disc brake squeal, 14th FISITA Congress, paper 1/9, 1972.

[7] Kinkaid N.M., O’Reilly O.M., Papadopoulos P., Automotive disc brake squeal, Journal of Sound and Vibration, 267, 105-166, 2003.

[8] Ouyang H., Nack W., Yuan Y., and Chen F.,. "Numerical analysis of automotive disc brake squeal : a review”. Int. J. Vehicle Noise, 1(3-4), 207-231, 2005.

[9] Massi F., Baillet L., Giannini O., Sestieri A., Brake squeal: Linear and nonlinear numerical approaches, Mechanical Systems and Signal Processing, 21, 23742393, 2007.

[10] Ouyang H., AbuBakar A.R., Complex eigenvalue analysis and dynamic transient analysis in predicting disc brake squeal, International Journal of Vehicle Noise and Vibration, 2 (2),143-155, 2006.

[11] Ouyang H., Mottershead J.E., Brookfields D.J., James S., Cartmell M.P., A methodology for the determination of dynamic instabilities in a car disc brake, International Journal of Vehicle Design, 23, 241-262, 2000.

[12]Fritz G., Sinou J.-J., Duffal J.-M., Jezequel L., Investigation of the relationship between damping and mode-coupling patterns in case of brake squeal, Journal of Sound and Vibration, 307, 591-609, 2007.

[13] Hoffmann N., Gaul L.,Effects of damping on mode-coupling instability in friction induced oscillations, ZAMM. Z. Angew. Math. Mech., 83(32), 847-866, 19631964.

[14] Fritz G., Sinou J.-J., Duffal J.-M., Jezequel L.,. Effects of damping on brake squeal coalescence patterns-application on a finite element model, Mechanics Research Communications, 34, 181-190, 2007.

[15] Cunefare A., Graf A.J., Experimental active control of automotive disc brake rotor squeal using dither, Journal of Sound and Vibration, 250(4),579-590, 2002. 